International Journal of Theoretical Physics 49 (2010) 293-303

\title{
Fractional Dynamics of Relativistic Particle
}

\author{
Vasily E.Tarasov \\ Skobeltsyn Institute of Nuclear Physics, \\ Moscow State University, Moscow 119991, Russia \\ E-mail: tarasov@theory.sinp.msu.ru
}

\begin{abstract}
Fractional dynamics of relativistic particle is discussed. Derivatives of fractional orders with respect to proper time describe long-term memory effects that correspond to intrinsic dissipative processes. Relativistic particle subjected to a non-potential four-force is considered as a nonholonomic system. The nonholonomic constraint in four-dimensional spacetime represents the relativistic invariance by the equation for four-velocity $u_{\mu} u^{\mu}+c^{2}=0$, where $c$ is a speed of light in vacuum. In the general case, the fractional dynamics of relativistic particle is described as non-Hamiltonian and dissipative. Conditions for fractional relativistic particle to be a Hamiltonian system are considered.
\end{abstract}

PACS 45.10.Hj;03.30.+p;45.20-d 


\section{Introduction}

The derivatives of non-integer orders are a natural generalization of the ordinary differentiation of integer order. Fractional differentiation with respect to time is characterized by long-term memory effects. The theory of derivatives and integrals of non-integer order goes back to Leibniz, Liouville, Riemann, Grunwald, and Letnikov [1, 2]. The interest in fractional equations [2, 3] has been growing continually during the last few years because of numerous applications in recent studies in mechanics and physics (for example, see books [4, 5, 6] and references therein).

In this paper, we discuss fractional dynamics of relativistic particles that are described as

nonholonomic systems in four-dimensional space-time. It is well known that components of the four-velocity $u^{\mu}=d x^{\mu} / d \tau(\mu=1,2,3,4$ and $\tau$ is a proper time $)$ are not independent. The components of the four-velocity are connected by the equation $u_{\mu} u^{\mu}+c^{2}=0$, where $c$ is a speed of light in vacuum. This equation allows us to consider the relativistic particle as a system with constraint in four-dimensional space-time. This constraint is nonlinear nonholonomic (nonintegrable) constraint. As a result, the relativistic invariance for point particles is represented by a nonholonomic constraint [7, 8, 9].

Note that only mechanics of relativistic particles can be considered as a mechanics with nonholonomic constraint. The relativistic invariance in the field theory cannot be represented as a nonholonomic constraint. At the same time, nonholonomic constraints can be used in the field theory. For example, the higher spin fields are connected with nonholonomic constraints [10] and the gauge fixing conditions for of non-abelian gauge fields can be described as nonholonomic constraints [11]. The Euler-Lagrange and Hamilton equations for nonholonomic systems in classical field theory are suggested in [12].

In the framework of the fractional dynamics, we consider a relativistic particle subjected to a general four-force. In the general case, the four-force is non-potential, and the relativistic particle is a non-Hamiltonian system in four-dimensional pseudo-Euclidean space-time. We consider fractional dynamics of non-Hamiltonian and dissipative systems in relativistic theory. The fractional equations of motion describes power-law memory effects that correspond 
to intrinsic dissipative processes in the relativistic systems. Note that relativistic particle with dissipation is discussed in [13, 14]. In Refs. [13, 14], the Lagrangian and Hamiltonian functions for one-dimensional relativistic particles with linear dissipation are suggested. In general, non-Hamiltonian and dissipative $n$-dimensional systems with $n>1$ cannot be described by Hamiltonian or Lagrangian since the Helmholtz's conditions for these systems are not satisfied [15]. In this paper, we consider fractional dynamics of relativistic particles as motions of four-dimensional non-Hamiltonian and dissipative systems.

In Sect. 2, the nonholonomic constraint in four-dimensional space-time for relativistic particle and some notations are considered. In Sect. 3, we discuss the fractional equations of motion for relativistic particle that is considered as a nonholonomic system. In Sect. 4, we discuss the d'Alembert-Lagrange principle for fractional equations of relativistic particle that is considered as a nonholonomic system. We prove that fractional equations for relativistic systems with nonholonomic constraint are represented as fractional equations for holonomic systems. In Sect. 5, the conditions for fractional relativistic particle to be a Hamiltonian or non-dissipative system are considered. Finally, a short conclusion is given in Sect. 6 .

\section{Nonholonomic Constraint}

We consider a four-dimensional pseudo-Euclidean space-time of points with coordinates $x^{\mu}$ : $x^{1}=x, x^{2}=y, x^{3}=z, x^{4}=c t$. The point coordinates in the four-dimensional space-time can be considered as components radius four-vector of the point particle, $\vec{R}=\left(x^{1}, x^{2}, x^{3}, x^{4}\right)=$ $(x, y, z, c t)$. The square of the elementary radius four-vector in the four-dimensional space-time is defined by $(d \vec{R})^{2}=\eta_{\mu \nu} d x^{\mu} d x^{\nu}$. Here and later we mean the sum on the repeated indices $\mu$ and $\nu$ from 1 to 4 . The coefficients $\eta_{\mu \nu}$ define a metric of pseudo-Euclidean space-time. This metric is a diagonal tensor such that $\eta_{11}=\eta_{22}=\eta_{33}=1$ and $\eta_{44}=-1$. Note that $x_{\mu}$ is not equal to $x^{\mu}$, since $x_{\mu}=\eta_{\mu \nu} x^{\nu}$ and $x_{1}=x^{1}, x_{2}=x^{2}, x_{3}=x^{3}$, and $x_{4}=-x^{4}$.

Assume that we have two radius four-vectors $\vec{R}$ and $\vec{R}^{\prime}$ with coordinates $x^{\mu}$ and $x^{\prime \mu}$ of two reference frames to describe a relativistic particle. If the coordinate transformation $x^{\prime \mu}=a^{\mu}{ }_{\nu} x^{\nu}$, 
where $a_{\nu}^{\mu}$ are constant values, satisfies the invariant condition:

$$
\left(d \vec{R}^{\prime}\right)^{2}=(d \vec{R})^{2}: \quad \eta_{\mu \nu} d x^{\prime \mu} d x^{\prime \nu}=\eta_{\alpha \beta} d x^{\alpha} d x^{\beta}
$$

then this transformation is a Lorenz transformation. The coordinates of the radius four-vector in the proper reference frame are $\vec{R}_{0}=(0,0,0, c \tau)$, where $\tau$ is a proper time. Condition (1) leads us to the relation

$$
(d \vec{R})^{2}=\left(d \vec{R}_{0}\right)^{2}: \quad \eta_{\mu \nu} d x^{\mu} d x^{\nu}=-c^{2} d \tau^{2}
$$

Using the definition of three-velocity $v^{k}=d x^{k} / d t, k=1,2,3$, we get

$$
d t=\gamma d \tau, \quad \gamma=\left(1-v^{2} / c^{2}\right)^{-1 / 2}
$$

Four-velocity of the point particle is defined as a derivative of the radius four-vector with respect to proper time:

$$
\vec{V}=\frac{d \vec{R}}{d \tau}: \quad u^{\mu}=\frac{d x^{\mu}}{d \tau}
$$

The components of the four-velocity $\vec{V}$ are $u^{k}=\frac{d x^{k}}{d \tau}=\gamma v^{k}, k=1,2,3$, and $u^{4}=\frac{d x^{4}}{d \tau}=c \gamma$. Note that rest particles $(\vec{v}=0)$ have $u^{4}=c$.

Equation (2) leads to the relation

$$
\left(\frac{d \vec{R}}{d \tau}\right)^{2}=\left(\frac{d \vec{R}_{0}}{d \tau}\right)^{2}: \quad \eta_{\mu \nu} \frac{d x^{\mu}}{d \tau} \frac{d x^{\nu}}{d \tau}=-c^{2}
$$

which means that square of the four-velocity is a constant value: $\vec{V}^{2}=-c^{2}$. Therefore we have the constraint equation

$$
\eta_{\mu \nu} u^{\mu} u^{\nu}+c^{2}=0
$$

As a result, a relativistic particle in the covariant formulation of relativistic mechanics is a system with the nonholonomic constraint. The constraint (4) is nonholonomic since it depends of velocity. Relativistic mechanics can be considered as nonholonomic mechanics in the fourdimensional space.

It is known that constraints in mechanics are some simplifications of real particle interactions. (Note that this statement is not correct in the field theory.) For example, if we consider 
the pendulum then we usually neglect of the forces of thread deformation. We also neglect of an interaction for constraint (44), which defines the relativistic invariance. If we use the nonholonomic constraint (44), then we neglect of a gravity interaction between particles. Let us consider the deformation of equations (2), (3) and (4) in general theory of relativity [16]. In the approximation of weak gravity fields, we have

$$
(d \vec{R})^{2}=\eta_{\mu \nu} d x^{\mu} d x^{\nu}-2 \varphi d t^{2}=-c^{2} d \tau^{2}
$$

where

$$
d t=\gamma^{\prime} d \tau, \quad \gamma^{\prime}=\left(1+\frac{2 \varphi}{c^{2}}-\frac{v^{2}}{c^{2}}\right)^{-1 / 2}
$$

and $\varphi$ is a classical (Newtonian) gravity potential. As a result, we have

$$
\eta_{\mu \nu} u^{\mu} u^{\nu}+c^{2}=2 \varphi \gamma^{\prime 2}
$$

Therefore nonholonomic constraint (4), which defines the relativistic invariance, is connected with the neglect of the gravity interaction, $\varphi=0$, (in general theory of relativity).

\section{Fractional equations of motion of relativistic particle}

Let $m_{0}$ be a rest mass of a point relativistic particle. The four-momentum of the particle is defined by $\vec{P}=m_{0} \vec{V}$. The components of the four-momentum are $p^{\mu}=m_{0} u^{\mu}$. Equation (4) gives

$$
\eta_{\mu \nu} p^{\mu} p^{\nu}+m_{0}^{2} c^{2}=0
$$

In relativistic mechanics the Newtonian equations are replaced by some generalization, which is invariant under the Lorenz transformations [17, 16]. The Newtonian equations are satisfied in the proper reference frame. The four-vector analog of the Newtonian equations is

$$
\frac{d \vec{P}}{d \tau}=\overrightarrow{\mathcal{F}}(\tau, \vec{R}, \vec{P})
$$

This equation is postulated as a main equation of relativistic dynamics. Equation (6) describes a relativistic particle subjected to a four-force $\overrightarrow{\mathcal{F}}=\overrightarrow{\mathcal{F}}(\tau, \vec{R}, \vec{P})$. Equation (6) must be considered 
with condition (51). As a result, we have the equations

$$
\frac{d x^{\mu}}{d \tau}=\frac{1}{m_{0}} p^{\mu}, \quad \frac{d p^{\mu}}{d \tau}=\mathcal{F}^{\mu}(\tau, x, p), \quad \eta_{\mu \nu} p^{\mu} p^{\nu}+m_{0}^{2} c^{2}=0 .
$$

If $d m_{0} / d \tau=0$, then (7) give

$$
m_{0} D_{\tau}^{2} x^{\mu}=\mathcal{F}^{\mu}(\tau, x, p)
$$

where $\eta_{\mu \nu} D_{\tau}^{1} x^{\mu} D_{\tau}^{1} x^{\nu}=-c^{2}$. These equations of motion can be generalized for fractional dynamics to take into account a power-law memory. We consider a generalization of (8) in the form of the fractional differential equations

$$
m_{0}{ }_{0}^{C} D_{\tau}^{\alpha} x^{\mu}=\mathcal{F}^{\mu}(\tau, x, p), \quad(1<\alpha<2)
$$

involving the Caputo fractional derivative ${ }_{0}^{C} D_{\tau}^{\alpha}$. The left-sided Caputo fractional derivative [2] of order $\alpha>0$ is defined by

$$
{ }_{0}^{C} D_{\tau}^{\alpha} x^{\mu}=\frac{1}{\Gamma(n-\alpha)} \int_{0}^{\tau} \frac{d \tau^{\prime} D_{\tau^{\prime}}^{n} x\left(\tau^{\prime}\right)}{\left(\tau-\tau^{\prime}\right)^{\alpha-n+1}}={ }_{0} I_{\tau}^{n-\alpha} D_{\tau}^{n} x^{\mu},
$$

where $n-1<\alpha<n, D_{\tau}^{n}=d^{n} / d \tau^{n}$, and ${ }_{0} I_{\tau}^{\alpha}$ is the left-sided Riemann-Liouville fractional integral

$$
{ }_{0} I_{\tau}^{\alpha} f(\tau)=\frac{1}{\Gamma(\alpha)} \int_{0}^{\tau} \frac{f\left(\tau^{\prime}\right) d \tau^{\prime}}{\left(\tau-\tau^{\prime}\right)^{1-\alpha}}, \quad(\tau>0) .
$$

Fractional derivative with respect to proper time describes a power-law memory effects that correspond to intrinsic dissipative processes.

Using $p^{\mu}=m_{0} D_{\tau}^{1} x^{\mu}$, equations (91) can be rewritten in the form

$$
\begin{gathered}
D_{\tau}^{1} x^{\mu}=\frac{1}{m_{0}} p^{\mu}, \\
{ }_{0}^{C} D_{\tau}^{\alpha-1} p^{\mu}=\mathcal{F}^{\mu}(\tau, x, p), \quad(1<\alpha<2),
\end{gathered}
$$

Fractional integration of (13) of order $\alpha-1$ gives

$$
{ }_{0} I_{\tau}^{\alpha-1}{ }_{0}^{C} D_{\tau}^{\alpha-1} p^{\mu}={ }_{0} I_{\tau}^{\alpha-1} \mathcal{F}^{\mu}(\tau, x, p)
$$

Using the fundamental theorem of fractional calculus [18]

$$
{ }_{0} I_{\tau}^{\alpha-1}{ }_{0}^{C} D_{\tau}^{\alpha-1} p^{\mu}=p^{\mu}(\tau)-p(0), \quad(0<1-\alpha<1),
$$


we obtain

$$
p^{\mu}(\tau)=p^{\mu}(0)+{ }_{0} I_{\tau}^{\alpha-1} \mathcal{F}^{\mu}(\tau, x, p)
$$

Differentiation of (15) gives

$$
D_{\tau}^{1} p^{\mu}={ }_{0} D_{\tau}^{2-\alpha} \mathcal{F}^{\mu}(\tau, x, p), \quad(0<2-\alpha<1)
$$

where ${ }_{0} D_{\tau}^{2-\alpha}$ is the left-sided Riemann-Liouville fractional derivative defined by

$$
{ }_{0} D_{\tau}^{\alpha} x^{\mu}=D_{\tau}^{n} I_{\tau}^{n-\alpha} x^{\mu}=\frac{1}{\Gamma(n-\alpha)} \frac{d^{n}}{d \tau^{n}} \int_{0}^{\tau} \frac{x\left(\tau^{\prime}\right) d \tau^{\prime}}{\left(\tau-\tau^{\prime}\right)^{\alpha-n+1}}, \quad(n-1<\alpha \leq n) .
$$

As a result, equation (9) is equivalent to the fractional equations

$$
\begin{gathered}
D_{\tau}^{1} x^{\mu}=\frac{1}{m_{0}} p^{\mu} \\
D_{\tau}^{1} p^{\mu}={ }_{0} D_{\tau}^{2-\alpha} \mathcal{F}^{\mu}(\tau, x, p), \quad(1<\alpha<2) .
\end{gathered}
$$

These equations describe fractional dynamics of relativistic particle. Fractional differentiation with respect to proper time is characterized by long-term memory effects that correspond to intrinsic dissipative processes in the relativistic systems.

\section{4 d'Alembert-Lagrange principle for fractional relativis- tic dynamics}

It is known that the general principle, which allows us to derive equations of motion with holonomic and nonholonomic constraints, is the d'Alembert-Lagrange principle. For equations (18) and (19) this principle leads to the variation equation

$$
\left(\frac{d p^{\mu}}{d \tau}-{ }_{0} D_{\tau}^{2-\alpha} \mathcal{F}^{\mu}(\tau, x, p)\right) \eta_{\mu \nu} \delta x^{\nu}=0
$$

Multiplying (19) on the variation $\delta x_{\mu}=\eta_{\mu \nu} \delta x^{\nu}$ and summing over $\mu$ we obtain this variational equation.

The variations of coordinates $\delta x^{\mu}, \mu=1, \ldots, 4$ are defined by the relation of the ideal constraint

$$
\mathcal{R}_{\mu} \delta x^{\mu}=0
$$


where $\mathcal{R}_{\mu}$ are components of the constraint force vector. The four-vector $\mathcal{R}_{\mu}$ can be considered as a contribution of the reaction associated with the constraint to the four-force ${ }_{0} D_{\tau}^{2-\alpha} \mathcal{F}^{\mu}(\tau, x, p)$. Because a reaction force does no work in a virtual movement that is consistent with the corresponding kinematical restriction, we conclude that $\mathcal{R}_{\mu}$ must be perpendicular to any $\delta x^{\mu}$ that satisfies the constraint equation. Thus, if $\delta x^{\mu}$ satisfies constraint equation, we have $\mathcal{R}_{\mu} \delta x^{\mu}=0$. We now consider which condition $\delta x^{\mu}$ must be realized in order to satisfy a constraint equations. We can derive the usual relativistic equations of motion only under the condition (21). For nonholonomic systems a definition of the variations was suggested by Chetaev [19, 20]. The variations $\delta x^{\mu}$ are defined by the condition:

$$
\frac{\partial f}{\partial u^{\mu}} \delta x^{\mu}=0
$$

where

$$
f=\eta_{\mu \nu} u^{\mu} u^{\nu}+c^{2}
$$

Using (21) and (22), we have the functions $\mathcal{R}_{\mu}$ as linear combinations of $\partial f / \partial u^{\mu}$, i.e.

$$
\mathcal{R}_{\mu}=\lambda \frac{\partial f}{\partial u^{\mu}}
$$

where $\lambda$ is a Lagrange multiplier. We note that substitution of (23) into (22) gives

$$
\eta_{\mu \nu} u^{\mu} \delta x^{\nu}=0
$$

Equations (20) and (22) give the variational equation

$$
\left(\frac{d p^{\mu}}{d \tau}-{ }_{0} D_{\tau}^{2-\alpha} \mathcal{F}^{\mu}(\tau, x, p)-\lambda \frac{\partial f}{\partial u^{\mu}}\right) \delta x^{\mu}=0 .
$$

This variational equation is equivalent to the fractional equations of motion

$$
\frac{d p^{\mu}}{d \tau}={ }_{0} D_{\tau}^{2-\alpha} \mathcal{F}^{\mu}(\tau, x, p)+\lambda \frac{\partial f}{\partial u^{\mu}} \quad(\mu=1,2,3,4)
$$

We cannot use constraint equation for the function $f$ in variational equation before the partial derivative on $u^{\mu}$ is taken.

Substitution of (23) into (25) gives the equations of motion

$$
\frac{d p^{\mu}}{d \tau}={ }_{0} D_{\tau}^{2-\alpha} \mathcal{F}^{\mu}(\tau, x, p)+2 \lambda u^{\mu}, \quad u_{\mu} u^{\mu}+c^{2}=0,
$$


where $p^{\mu}=m_{0} u^{\mu}$ and $u^{\mu}=d x^{\mu} / d \tau$. The system of equations (26) is a closed system of five equations in the same number of unknowns $x^{\mu}$ and $\lambda$. Using these equations, we can find the multiplier $\lambda$ as a function $\lambda=\lambda(\tau, x, p)$. Substituting this function in (25), we get the equations for coordinates $x^{\mu}$. It allows us to represent the fractional equations of motion for relativistic systems with nonholonomic constraint as fractional equations for holonomic systems.

Differentiating of constraint (5) with respect to $\tau$, we obtain

$$
\eta_{\mu \nu} \frac{d p^{\mu}}{d \tau} p^{\nu}+m_{0} \frac{d m_{0}}{d \tau} c^{2}=0
$$

Substituting (27) in (26) with $m_{0} u^{\mu}=p^{\mu}$ and $d m_{0} / d \tau=0$, we get

$$
\eta_{\mu \nu} p_{0}^{\nu} D_{\tau}^{2-\alpha} \mathcal{F}^{\mu}(\tau, x, p)+2 \lambda \eta_{\mu \nu} u^{\mu} p^{\nu}=0
$$

Using the constraint equation $\eta_{\mu \nu} u^{\mu} u^{\nu}=-c^{2}$ and the four-momentum $p^{\mu}=m_{0} u^{\mu}$, we obtain the Lagrange multiplier

$$
\lambda=\frac{1}{2 c^{2}}\left(\eta_{\mu \nu} u_{0}^{\mu} D_{\tau}^{2-\alpha} \mathcal{F}^{\nu}(\tau, x, p)\right) .
$$

Therefore the reaction four-force $\mathcal{R}^{\mu}$ of the nonholonomic constraint is

$$
\mathcal{R}^{\mu}=2 \lambda u^{\mu}=\frac{1}{c^{2}} u^{\mu}\left(u_{\nu 0} D_{\tau}^{2-\alpha} \mathcal{F}^{\nu}\right)
$$

As a result, we have the fractional equation

$$
\frac{d p^{\mu}}{d \tau}={ }_{0} D_{\tau}^{2-\alpha} \mathcal{F}^{\mu}(\tau, x, p)+\frac{1}{c^{2}} u^{\mu}\left(u_{\nu 0} D_{\tau}^{2-\alpha} \mathcal{F}^{\nu}\right)
$$

These equations define a holonomic system subjected to the sum of four-forces ${ }_{0} D_{\tau}^{2-\alpha} \mathcal{F}^{\mu}+\mathcal{R}^{\mu}$. If initial dates satisfy constraint equation (44), then the solution of Eq. (28) describes a fractional dynamics of the relativistic point particle as a holonomic system.

As a result, we prove the following statement.

Proposition. Fractional equations for the relativistic particle subjected to a non-potential four-force $\mathcal{F}^{\mu}$, which have the form

$$
\frac{d x^{\mu}}{d \tau}=\frac{1}{m_{0}} p^{\mu}, \quad \frac{d p^{\mu}}{d \tau}={ }_{0} D_{\tau}^{2-\alpha} \mathcal{F}^{\mu}(\tau, x, p), \quad \eta_{\mu \nu} p^{\mu} p^{\nu}+m_{0}^{2} c^{2}=0
$$


with $d m_{0} / d \tau=0$, are equivalent to the equations

$$
\frac{d x^{\mu}}{d \tau}=\frac{1}{m_{0}} p^{\mu}, \quad \frac{d p^{\mu}}{d \tau}={ }_{0} D_{\tau}^{2-\alpha} \mathcal{F}^{\mu}(\tau, x, p)+\mathcal{R}^{\mu}(\tau, x, p)
$$

where

$$
\mathcal{R}^{\mu}(\tau, x, p)=\frac{1}{m_{0}^{2} c^{2}} p^{\mu}\left(p_{\nu 0} D_{\tau}^{2-\alpha} \mathcal{F}^{\nu}\right)
$$

and the initial dates satisfy constraint condition (4).

The solution of Eq. (30) describes the fractional dynamics of the relativistic particle.

\section{Fractional non-Hamiltonian and dissipative relativis- tic systems}

The system is called locally Hamiltonian if the sum of applied forces satisfies the Helmholtz conditions [21, 22]. If $(x, p) \in \mathcal{M}$ and $\mathcal{M}$ is a simply connected region, then a locally Hamiltonian system is globally Hamiltonian. A region is simply connected if it is path-connected and every path between two points can be continuously transformed into every other. A region where any two points can be joined by a path is called path-connected.

The Helmholtz conditions for fractional equations (30) have the form

$$
\begin{gathered}
\frac{\partial\left({ }_{0} D_{\tau}^{2-\alpha} \mathcal{F}^{\mu}\right)}{\partial p^{\nu}}+\frac{\partial \mathcal{R}^{\mu}}{\partial p_{\nu}}=0, \\
\frac{\partial\left({ }_{0} D_{\tau}^{2-\alpha} \mathcal{F}^{\mu}\right)}{\partial x^{\nu}}+\frac{\partial \mathcal{R}^{\mu}}{\partial x^{\nu}}-\frac{\partial\left({ }_{0} D_{\tau}^{2-\alpha} \mathcal{F}^{\nu}\right)}{\partial x^{\mu}}-\frac{\partial \mathcal{R}^{\nu}}{\partial x^{\mu}}=0 .
\end{gathered}
$$

Substitution of (31) into Eqs. (32) and (33) gives

$$
\begin{gathered}
\frac{\partial\left({ }_{0} D_{\tau}^{2-\alpha} \mathcal{F}^{\mu}\right)}{\partial p^{\nu}}+\frac{1}{m_{0}^{2} c^{2}} \frac{\partial\left[p^{\mu}\left(p_{\sigma 0} D_{\tau}^{2-\alpha} \mathcal{F}^{\sigma}\right)\right]}{\partial p^{\nu}}=0, \\
\frac{\partial\left({ }_{0} D_{\tau}^{2-\alpha} \mathcal{F}^{\mu}\right)}{\partial x^{\nu}}+\frac{1}{m_{0}^{2} c^{2}} p^{\mu}\left(p_{\sigma} \frac{\partial\left({ }_{0} D_{\tau}^{2-\alpha} \mathcal{F}^{\sigma}\right)}{\partial x^{\nu}}\right)- \\
-\frac{\partial\left({ }_{0} D_{\tau}^{2-\alpha} \mathcal{F}^{\nu}\right)}{\partial x^{\mu}}-\frac{1}{m_{0}^{2} c^{2}} p^{\nu}\left(p_{\sigma} \frac{\partial\left({ }_{0} D_{\tau}^{2-\alpha} \mathcal{F}^{\sigma}\right)}{\partial x^{\mu}}\right)=0 .
\end{gathered}
$$


These equations are the Helmholtz conditions [21, 22] for fractional relativistic dynamics. If these conditions are satisfied then the fractional dynamics of relativistic particle is Hamiltonian. The fractional relativistic particle subjected to a four-force $\mathcal{F}^{\mu}(\tau, x, p)$ is non-Hamiltonian if the Helmholtz conditions (34) and (35) are not satisfied [15].

If

$$
\Omega(x, p)=\sum_{\mu=1}^{4}\left(\frac{\partial\left({ }_{0} D_{\tau}^{2-\alpha} \mathcal{F}^{\mu}\right)}{\partial p^{\mu}}+\frac{\partial\left({ }_{0} D_{\tau}^{2-\alpha} \mathcal{R}^{\mu}\right)}{\partial p^{\mu}}\right) \neq 0,
$$

then we have a generalized dissipative system [15]. If $\Omega(x, p) \leq 0$ for all points $(x, p)$ and $\Omega(x, p)<0$ for some points $(x, p)$, then the system is a dissipative system.

Note that a one-dimensional relativistic particle with dissipation is considered in Refs. [13, 14. The Lagrangian and Hamiltonian functions for one-dimensional relativistic particles with linear dissipation are suggested. In general, non-Hamiltonian and dissipative $n$-dimensional systems with $n>1$ cannot be described by Hamiltonian or Lagrangian since the Helmholtz's conditions for these systems are not satisfied [15].

In fractional relativistic dynamics the principle of stationary action for particle subjected to non-potential forces $\mathcal{F}^{\mu}(\tau, x, p)$ can be used if the Helmholtz conditions (34) and (35) are satisfied. The Hamilton's principle and the principle of stationary action are equivalent only for special forms of the four-force $\mathcal{F}^{\mu}(\tau, x, p)$. We note that the Hamilton's principle is described by nonholonomic variational equation [23, 24, 25, 26, 27, 28]. It allows us to use this principle to obtain fractional equations of motion for non-Hamiltonian and dissipative systems. The principle of stationary action is defined by holonomic variational equation. Therefore the principle of stationary action cannot be to derive fractional equations of motion in the general case. In general, the Hamilton's principle and nonholonomic variational equations can be used to describe fractional dynamics of relativistic systems. We note that the fractional equations of motion which follow from the d'Alembert-Lagrange principle are not equivalent to the fractional equations which follow from the principle of stationary action. In Refs. [23, 24, 25, 29, 30], authors give proofs that the solutions to the equations of motion which follow from the d'AlembertLagrange principle and the Hamilton's principle do not in general satisfy the equations which follow from the action principle with nonholonomic constraints. The variational Sedov's equa- 
tion [26, 27, 28] (see also [31, 32]) can be used in fractional relativistic dynamics instead of the principle of stationary action. We note that relativistic models of continuous media with dissipation are considered in [28, 32].

Let us consider the four-vector ${ }_{0} D_{\tau}^{2-\alpha} \mathcal{F}^{\mu}$ as the sum

$$
{ }_{0} D_{\tau}^{2-\alpha} \mathcal{F}^{\mu}=G^{\mu}+\Pi^{\mu},
$$

where $\left(G^{\mu} u_{\mu}\right)=0$, and $\left(\Pi^{\mu} u_{\mu}\right) \neq 0$. Substitution of (36) into (30) of the form (28) gives

$$
\frac{d p^{\mu}}{d \tau}=G^{\mu}+\Pi^{\mu}+\frac{1}{c^{2}} u^{\mu}\left(\Pi^{\nu} u_{\nu}\right)
$$

The four-force $G^{\mu}$ is usually called [17] a real mechanical force, which satisfies the orthogonal condition $u_{\mu} G^{\mu}=0$. The four-vector $\Pi^{\mu}$ describes the energy-momentum exchange between the point particle and medium. The components of $\Pi^{\mu}$ are

$$
\Pi^{\mu}=(\gamma \vec{\Pi},(\gamma / c) \Phi)
$$

where $\vec{\Pi}$ and $\Phi$ are momentum and energy, which are transmitted by convection per unit time. For the heat transfer, three-momentum $\delta \vec{p}$ and energy $\delta Q$ transmitted per time $d \tau$ are defined by the formulas $\delta \vec{p}=\vec{\Pi} d t$, and $\delta Q=\Phi d t$. The components of $\delta Q^{\mu}$ are

$$
\delta Q^{\mu}=\Pi^{\mu} d \tau=\left(\delta \vec{p}, \frac{1}{c} \delta Q\right)=\left(\gamma \vec{\Pi} d \tau, \frac{\gamma}{c} \Phi d \tau\right),
$$

where $\delta Q^{\mu}$ is a four-vector of the heat energy-momentum, which is transmitted per time $d \tau$. Note that the value $-u_{\mu} \Pi^{\mu}=-\gamma^{2}((\vec{\Pi}, \vec{v})-\Phi)$, is a velocity of the convective transmission of incoming energy in the rest reference frame. The four-vectors $G^{\mu}$ and $\Pi^{\mu}$ allow us to describe non-Hamiltonian and dissipative processes in fractional relativistic mechanics.

\section{Conclusion}

We formulate fractional dynamics of relativistic point particle as mechanics of the systems with nonholonomic constraint in the four-dimensional pseudo-Euclidean space-time. We consider fractional dynamics of relativistic particles subjected to four-forces that can be non-potential. 
The conditions on the four-forces that allow us to consider fractional dynamics of relativistic particles subjected to non-potential forces as Hamiltonian dynamics are suggested. We prove that the nonholonomic constraint, which represents relativistic invariance, and the nonpotential four-force can be compensated such that the fractional dynamics is Hamiltonian (and non-dissipative).

Let us note some possible extensions of the fractional relativistic dynamics.

1. Nonholonomic constraints with power-law memory [33], which are described by fractional equations, can be considered in relativistic mechanics by using fractional derivatives [2] with respect to proper time.

2. The suggested fractional relativistic dynamics can be used to generalize quantum theory of non-Hamiltonian and dissipative systems [15].

3. In the framework of the fractional relativistic dynamics it is possible to consider a relativistic generalization of the fractional variational problems [34, 35] in Lagrangian and Hamiltonain form [36, 37, 38, 39, 40. Note that nonholonomic variational equations must be used since the fractional equations which follow from the d'Alembert-Lagrange principle (and the Hamilton's principle) do not in general equivalent the equations which follow from the action principle with nonholonomic constraints [23, 24, 25, 29, 30].

The study of plasma systems containing ensembles of particles (dust) is a rapidly developing field of complex systems research. One of the general features of complex plasma systems is the presence of non-potential interaction forces between the dust particles due to the dynamic interaction between the dust particles and the plasma (for example, see [41, 42, 43] and references therein). In general, these systems cannot be described as Hamiltonian, since the energy is not conserved because of the openness of the systems due to plasma-particle interaction. We hope that fractional dynamics of relativistic particle subjected to non-potential forces can be used to describe relativistic complex plasma systems. 


\section{References}

[1] S.G. Samko, A.A. Kilbas, O.I. Marichev, Fractional Integrals and Derivatives Theory and Applications (Gordon and Breach, New York, 1993).

[2] A.A. Kilbas, H.M. Srivastava, J.J. Trujillo, Theory and Application of Fractional Differential Equations (Elsevier, Amsterdam, 2006).

[3] I. Podlubny, Fractional Differential Equations (Academic Press, San Diego, 1999).

[4] G.M. Zaslavsky, Hamiltonian Chaos and Fractional Dynamics (Oxford University Press, Oxford, 2005).

[5] A. Carpinteri, F. Mainardi, (Eds), Fractals and Fractional Calculus in Continuum Mechanics (Springer, Wien, 1997).

[6] J. Sabatier, O.P. Agrawal, J.A. Tenreiro Machado, (Eds.), Advances in Fractional Calculus. Theoretical Developments and Applications in Physics and Engineering (Springer, Dordrecht, 2007)

[7] O. Krupkova, J. Musilova, "The relativistic particle as a mechanical system with nonholonomic constraints" Journal of Physics A 34(18), (2001) 3859-3875.

[8] O. Krupkova, J. Musilova, "The relativistic mechanics in a nonholonomic setting: A unified approach to particles with non-zero mass and massless particles" (arXiv:0904.2933)

[9] X. Gracia, R. Martin, "Regularity and symmetries of nonholonomic systems" Journal of Physics A 38(5), (2005) 1071-1087. (Section 6. pp.1081-1083) math-ph/0405066

[10] E.C.G. Sudarshan, "Higher spin fields and non-holonomic constraints" Foundations of Physics, 33 (5), (2003) 707-717.

[11] H. Nakagoshi, M. Namiki, I. Ohba, K. Okano, "Gauge fixing condition as non-holonomic constraint in stochastic quantization of non-abelian gauge fields" Progress of Theoretical Physics 70 (1), (1983) 326-329.

[12] O. Krupkova, P. Voln, "Euler-Lagrange and Hamilton equations for non-holonomic systems in field theory" Journal of Physics A 38 (2005) 8715-8745.

[13] G. Gonzalez, "Hamiltonian for a relativistic particle with linear dissipation" International Journal of Theoretical Physics 46(3), (2007) 486-491. 
[14] G. Gonzalez, "Relativistic motion with linear dissipation" International Journal of Theoretical Physics 46(3), (2007) 417-423. (arXiv:quant-ph/0503211)

[15] V.E. Tarasov, Quantum Mechanics of Non-Hamiltonian and Dissipative Systems (Elsevier Science, Amsterdam, New York, 2008).

[16] W. Pauli, Theory of Relativity (Pergamon Press, 1958) or (Dover, 1981).

[17] V.A. Ugarov, Special Theory of Relativity (MIR Publishers, Moscow, 1979) 406p; and 2-nd ed. (Nauka, Moscow, 1997) 384p. pp.141-142. in Russian.

[18] V.E. Tarasov, "Fractional vector calculus and fractional Maxwell's equations" Annals of Physics 323(11), (2008) 2756-2778. (arXiv:0907.2363)

[19] N.G. Chetaev, "About Gauss Principle" in Proc. Phys. Math. Soc. of Kazan' Univer. 6 Ser.3. (1932-1933) 68-71.

[20] N.G. Chetaev, Stability of Motion. Works on Analytic Mechanics (Acad. Sci. USSR, Moscow, 1962) pp.323-326.

[21] H. Helmholtz, J. Reine Angew. Math. 100 (1886) 137-166.

[22] V.E. Tarasov, "Fractional generalization of gradient and Hamiltonian systems" Journal of Physics A 38 (26), (2005) 5929-5943. (arXiv:math/0602208)

[23] V.V. Rumiantsev, "On Hamilton's principle for nonholonomic systems" Journal of Applied Mathematics and Mechanics 42(3), (1978) 407-419.

[24] V. V. Rumyantsev, "Hamilton's principle for nonholonomic systems" Prikladnaya Matematika i Mekhanika 42(3), (1978) 387-399 in Russian.

[25] V.V. Rumyantsev, "Forms of Hamiltons principle for nonholonomic systems" Facta Universitatis. Series Mechanics, Automatic Control and Robotics 2(19), (2000) 1035-1048. http://facta.junis.ni.ac.rs/macar/macar2000/macar2000-02.pdf

[26] L.I. Sedov, "Models of continuous media with internal degrees of freedom" Journal of Applied Mathematics and Mechanics 32(5), (1968) 803-819; Prikladnaya Matematika i Mekhanika 32 (5), (1968) 771-785. in Russian.

[27] L.I. Sedov, "Mathematical methods for constructing new models of continuous media" Russian Mathematical Surveys 20(5), (1965) 123-182. 
[28] L.I. Sedov, A.G. Tsypkin, Principles of the Microscopic Theory of Gravitation and Electromagnetism (Nauka, Moscow, 1989) in Russian, Sec. 3.7, and Sec. 3.8-3.12, Sec. 4.

[29] V.V. Rumiantsev, "On integral principles for nonholonomic systems" Journal of Applied Mathematics and Mechanics 46(1), (1982) 1-8.

[30] C. Cronström, T. Raita, "On nonholonomic systems and variational principles" Journal of Mathematical Physics 50 (4), (2009) 042901. (arXiv:0810.3611)

[31] V.E Tarasov, "Fractional variations for dynamical systems: Hamilton and Lagrange approaches" Journal of Physics A 39 (26), (2006) 8409-8425. (arXiv:math-ph/0606048)

[32] L.T. Chernyi, Relativistic Models of Continuous Media (Nauka, Moscow, 1983) in Russian.

[33] V.E. Tarasov, G.M. Zaslavsky, "Nonholonomic constraints with fractional derivatives" Journal of Physics A 39(31), (2006) 9797-9815. (arXiv:math-ph/0603067)

[34] O.P. Agrawal, "Formulation of Euler-Lagrange equations for fractional variational problems" J. Math. Anal. Appl. 272 (2002) 368-379.

[35] O.P. Agrawal, "Fractional variational calculus in terms of Riesz fractional derivatives" J. Phys. A. 40 (2007) 6287-6303.

[36] F. Riewe, "Nonconservative Lagrangian and Hamiltonian mechanics" Physical Review E 53 (1996) 1890-1899.

[37] M. Klimek, "Lagrangean and Hamiltonian fractional sequential mechanics" Czechoslovak Journal of Physics 52(11), (2002) 1247-1253.

[38] S. I. Muslih, D. Baleanu, "Formulation of Hamiltonian equations for fractional variational problems" Czechoslovak Journal of Physics 55 (2005) 633-642.

[39] E.M. Rabei, K.I. Nawafleh, R.S. Hijjawi, S.I. Muslih, D. Baleanu, "The Hamilton formalism with fractional derivatives" J. Math. Anal. Appl. 327(2), (2007) 891-897.

[40] M.A.E. Herzallah, D. Baleanu, "Fractional-order Euler-Lagrange equations and formulation of Hamiltonian equations" Nonlinear Dynamics 58(1-2), (2009) 385-391.

[41] R. Kompaneets, S.V. Vladimirov, A.V. Ivlev, V. Tsytovich, G. Morfill, "Dust clusters with non-Hamiltonian particle dynamics" Physics of Plasmas 13(7), (2006) 072104. 
[42] J.D.E. Stokes, S.V. Vladimirov, A.A. Samarian, "Reformulation of Hamiltonian dynamics for dust particle interactions in complex plasma" 34th EPS Conference on Plasma Physics. Warsaw, 2-6 July 2007 ECA Vol.31F, O-2.018 (2007) http://epsppd.epfl.ch/Warsaw/pdf/O2_018.pdf

[43] S.K. Zhdanov, A.V. Ivlev, G.E. Morfill, "Non-Hamiltonian dynamics of grains with spatially varying charges" Physics of Plasmas 12(2), (2005) 072312.

[44] D. Baleanu, A.K. Golmankhaneh, "The dual action of fractional multi-time Hamilton equations" International Journal of Theoretical Physics 48(9), (2009) 2558-2569. 